\title{
EDITORIAL
}

\section{Interleukin-6: a local pain trigger?}

\author{
Camilla I Svensson \\ See related research by Boettger et al., http://arthritis-research.com/content/12/4/R140
}

\begin{abstract}
Pain management in conditions of chronic

inflammation is a clinical challenge, and increasing our

understanding of the mechanisms driving this type

of pain is important. In the previous issue of Arthritis

Research \& Therapy, Boettger and colleagues examine

the role of IL- 6 in antigen-induced arthritis using the

IL-6 neutralizing soluble glycoprotein 130 and link IL-6

to a pathophysiological role in the generation of pain, independent of the proinflammatory properties of IL-6.

The findings presented in this study add to a growing

body of evidence highlighting the role of IL-6 in the

induction and maintenance of pain.
\end{abstract}

Adequate pain management in conditions of chronic inflammation, such as rheumatoid arthritis, is a clinical challenge. While pain-relieving drugs such as cyclooxygenase-inhibitors and opioids work well in the short term, long-term use is frequently associated with side effects. The ongoing search for novel drugs for treatment of chronic pain is therefore well motivated. Proinflammatory cytokines such as TNF, IL-1 and IL- 6 are major mediators of joint inflammation and destruction, and an increasing pool of preclinical data indicates that these cytokines are also key players in the generation and maintenance of pain. In the previous issue of Arthritis Research \& Therapy, Boettger and colleagues focus on IL-6 and report that this cytokine plays a significant role in arthritis-induced joint pain [1].

IL-6 is a multifunctional cytokine, with proinflammatory and other regulatory properties. IL-6 exerts its effect on target cells by binding to the IL- 6 specific membrane receptor (IL-6R), which activates the transducer glycoprotein 130 (gp130), the second subunit of the IL-6R complex. Neurons are devoid of membrane-bound IL-6R, but these cells can still react to IL- 6 when IL- 6 is bound

*Correspondence: camilla.svensson@ki.se

Department of Physiology and Pharmacology, Karolinska Institutet, Von Eulers vag 8,17177 Stockholm, Sweden to the soluble form of IL-6R. The IL-6/soluble IL-6R complex activates gp130, which is expressed on most cells, including neurons. While the functional role of gp130 expressed on neurons is not well understood, this protein has been linked to sensitization of pain-sensing nerves through activation of phosphoinositide 3-kinase, protein kinase C-delta and Janus kinase/signal transducer and activator of transcription 3 signaling pathways and through regulation of the pain-activated ion channel TRPV1 [2,3]. Using soluble glycoprotein 130 (sgp130), which binds and inactivates IL-6, Boettger and colleagues made several important findings utilizing a rat model of antigen-induced arthritis.

First, the authors report that intraarticular administration of sgp130 attenuates arthritis-induced pain. This is an important finding as it suggests that IL-6 is a pain mediator - not only following nerve injury and tumor growth as has been reported earlier [2,3], but also subsequent to inflammatory arthritis. The pain-relieving (antinociceptive) effect of local versus systemic injections of sgp130 was investigated, and, surprisingly, intraarticular injection of gp130 to the knee joint had a greater antinociceptive effect than intraperitoneal injections. This may be interpreted as local IL- 6 in the joint being crucial for the nociceptive response, while circulating IL-6 is not. In such a case, one would expect a higher dose of systemic spg130 than used in Boettger and colleagues' study to have similar pain-relieving effects, given that it reaches the joint in high enough concentrations.

From a clinical perspective it is critical that a painrelieving drug has the ability to reverse alreadyestablished hypersensitivity, particularly in conditions such as arthritis where pain often is what brings the patient to seek medical attention. In the work presented by Boettger and colleagues, intraperitoneal post-treatment only decreased stimulus-evoked pain responses while intraarticular pretreatment had a broad antinociceptive effect, also normalizing weight bearing, locomotion and gait - measures thought to indirectly reflect ongoing pain. Because the routes of administration were different in the pretreatment versus post-treatment comparison (intraarticular versus intraperitoneal, respectively), further studies are warranted to determine 
whether IL-6 inhibition is antinociceptive when administrated during active arthritis. This determination is particularly important from the aspect of the antigeninduced arthritis model being predictive of the human condition, as clinical studies have shown that rheumatoid arthritis patients treated with monoclonal IL-6R antibody score lower on the pain visual analog scale and report a reduced number of tender joints [4].

Another noteworthy finding is that while one injection of sgp130 into the joint had an antinociceptive effect that lasted several days, no concurrent reduction of the joint inflammation was observed. Blocking IL-6-mediated signaling, even in the presence of other factors that continuously drive the inflammatory process, is therefore sufficient to bring the nociceptive thresholds back towards normal levels. In support of this notion, it has been demonstrated that IL-6 can trigger activation of pain fibers in the absence of inflammation. Exposure to IL-6 sensitizes both peripheral and spinal sensory neurons by electrophysiological measures [5-7], and application of IL-6 to the peripheral nerve or into the cerebrospinal fluid evokes long-lasting pain behavior $[7,8]$, indicating that the IL6/soluble IL-6R complex may be acting directly on neurons. This does not exclude, however, the fact that IL-6 is also involved in the regulation of pain processing through actions on nonneuronal cells. For example, IL-6 is elevated in the spinal cord subsequent to peripheral nerve injury in rats, which has been linked to microglia activation and associated neuropathic pain $[3,8]$.

Finally, Boettger and colleagues found that although sgp130 failed to attenuate joint inflammation, bone erosion was reduced in the antigen-induced arthritis model. The structure-sparing effect of sgp130 is in line with recent work showing that IL-6R inhibition blocks osteoclast formation in vitro and in vivo, independent of the anti-inflammatory effect [9], and that bone erosion is reduced in rheumatoid arthritis patients on IL-6 inhibitor monotherapy [10]. The antinociceptive effect observed in the study by Boettger and colleagues was therefore possibly at least partly related to a prevention of joint destruction. This observation highlights the need for gaining a better and more detailed understanding of how bone erosion affects the sensory system.

In summary, there are several potential sites and mechanisms through which IL-6 may drive pain in conditions such as rheumatoid arthritis. While there are still critical steps to take before adding IL- 6 blockers to the list of painkillers, it is an intriguing thought that anticytokine therapies have the potential to become new tools for treatment of chronic pain.

\section{Abbreviations}

gp130, glycoprotein 130; IL, interleukin; IL-6R, IL-6 membrane receptor; sgp130, soluble glycoprotein 130; TNF, tumor necrosis factor; TRPV1, transient receptor potential cation channel, subfamily Vanilloid, member 1.

\section{Competing interests}

The author declares that she has no competing interests.

\section{Acknowledgements}

The present work was supported by the Swedish Research Council and the European Union Marie Curie International Reintegration Grant.

Published: 28 October 2010

\section{References}

1. Boettger MK, Leuchtweis J, Kummel D, Gajda M, Brauer R, Schaible HG: Differential effects of locally and systemically administered soluble glycoprotein 130 on pain and inflammation in experimental arthritis. Arthritis Res Ther 2010, 12:R140.

2. Andratsch M, Mair N, Constantin CE, Scherbakov N, Benetti C, Quarta S, Vogl C, Sailer CA, Uceyler N, Brockhaus J, Martini R, Sommer C, Zeilhofer HU, Müller W, Kuner R, Davis JB, Rose-John S, Kress M: A key role for gp130 expressed on peripheral sensory nerves in pathological pain. J Neurosci 2009, 29:13473-13483.

3. Dominguez E, Rivat C, Pommier B, Mauborgne A, Pohl M: JAK/STAT3 pathway is activated in spinal cord microglia after peripheral nerve injury and contributes to neuropathic pain development in rat. J Neurochem 2008, 107:50-60.

4. Smolen JS, Beaulieu A, Rubbert-Roth A, Ramos-Remus C, Rovensky J, Alecock E, Woodworth T, Alten R: Effect of interleukin-6 receptor inhibition with tocilizumab in patients with rheumatoid arthritis (OPTION study): a double-blind, placebo-controlled, randomised trial. Lancet 2008, 371:987-997.

5. Kawasaki Y, Zhang L, Cheng JK, Ji RR: Cytokine mechanisms of central sensitization: distinct and overlapping role of interleukin-1 $\beta$, interleukin- 6 , and tumor necrosis factor-a in regulating synaptic and neuronal activity in the superficial spinal cord. J Neurosci 2008, 28:5189-5194.

6. Brenn D, Richter F, Schaible HG: Sensitization of unmyelinated sensory fibers of the joint nerve to mechanical stimuli by interleukin- 6 in the rat: an inflammatory mechanism of joint pain. Arthritis Rheum 2007, 56:351-359.

7. Eliav E, Benoliel R, Herzberg U, Kalladka M, Tal M: The role of IL-6 and IL-1 $\beta$ in painful perineural inflammatory neuritis. Brain Behav Immun 2009, 23:474-484.

8. Lee KM, Jeon SM, Cho HJ: Interleukin-6 induces microglial CX3CR1 expression in the spinal cord after peripheral nerve injury through the activation of p38 MAPK. Eur J Pain 2010, 14:682.e1-e12.

9. Axmann R, Bohm C, Kronke G, Zwerina J, Smolen J, Schett G: Inhibition of interleukin- 6 receptor directly blocks osteoclast formation in vitro and in vivo. Arthritis Rheum 2009, 60:2747-2756.

10. Nishimoto N, Hashimoto J, Miyasaka N, Yamamoto K, Kawai S, Takeuchi T, Murata N, van der Heijde D, Kishimoto T: Study of active controlled monotherapy used for rheumatoid arthritis, an IL-6 inhibitor (SAMURAI): evidence of clinical and radiographic benefit from an $X$ ray reader-blinded randomised controlled trial of tocilizumab. Ann Rheum Dis 2007, 66:1162-1167.

doi:10.1186/ar3138

Cite this article as: Svensson Cl: Interleukin-6: a local pain trigger? Arthritis Research \& Therapy 2010, 12:145. 